\title{
Charged-particle flow measured with the KRATTA detector in the ASY-EOS experiment
}

\author{
S. Kupny ${ }^{1}$, J. BrzyChCZYK ${ }^{1}$, J. EukAsiK ${ }^{2}$, P. PAwŁOWski ${ }^{1}$ and \\ the ASY-EOS COLLABORATION \\ ${ }^{1}$ Institute of Physics, Jagiellonian University, Kraków, Poland \\ ${ }^{2}$ Institute of Nuclear Physics, PAN, Kraków, Poland
}

\begin{abstract}
The flow of light charged particles from the $\mathrm{Au}+\mathrm{Au}$ reaction at $400 \mathrm{AMeV}$ measured with the KRATTA detector is presented. The results are compared with the FOPI data.
\end{abstract}

The KRATTA detector (Krakow Triple Telescope Array) [1] has been used to measure the energy, emission angles and isotopic composition of light charged reaction products in the ASY-EOS experiment at GSI laboratory [2].

This versatile, low threshold, broad energy range system consists of 38 independent modules which can be arranged in an arbitrary configuration Fig. 1 presents the used $7 \times 5$ array. A single module, covering actively about $4.5 \mathrm{msr}$ of the solid angle at the optimal distance of $40 \mathrm{~cm}$ from the target, consists of three identical, $500 \mu \mathrm{m}$ thick, large area photodiodes, used also for direct detection, and of two CsI (1500 ppm Tl) crystals of 2.5 and 12.5 $\mathrm{cm}$ length, respectively. The presented results come from the analysis of the experimental data for $\mathrm{Au}+\mathrm{Au}$ at $400 \mathrm{MeV} /$ nucleon incident energy.

Signals from the KRATTA modules have been stored for the off-line analysis using the $100 \mathrm{MHz}$ V1724 CAEN digitizers. The pulse shape analysis allowed to decompose the complex signals from the middle photodiode (Single Chip Telescope [3] segment, SCT) into the ionization and scintil- 


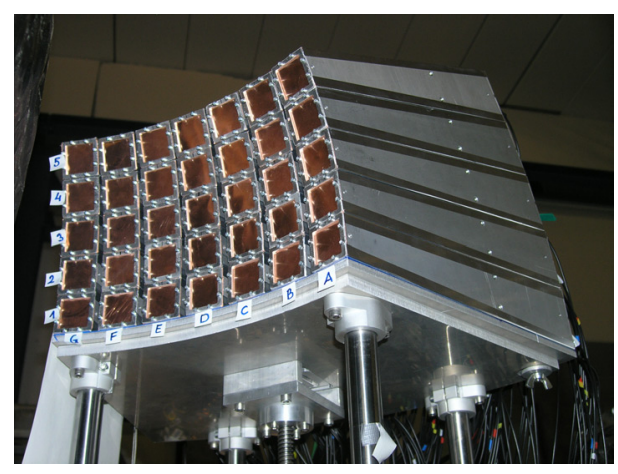

Figure 1: KRATTA detector during the ASY-EOS experiment (May 2011).

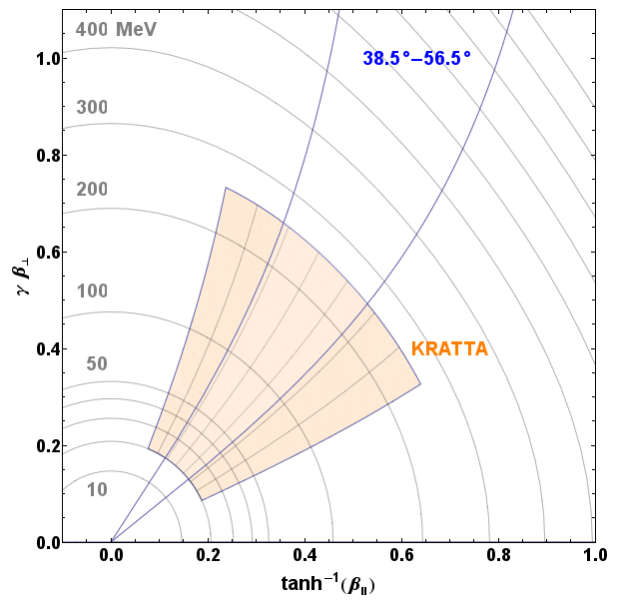

Figure 2: Acceptance for protons and angular range used for comparisons with FOPI.

lation components and to obtain a satisfactory isotopic resolution with a single readout channel.

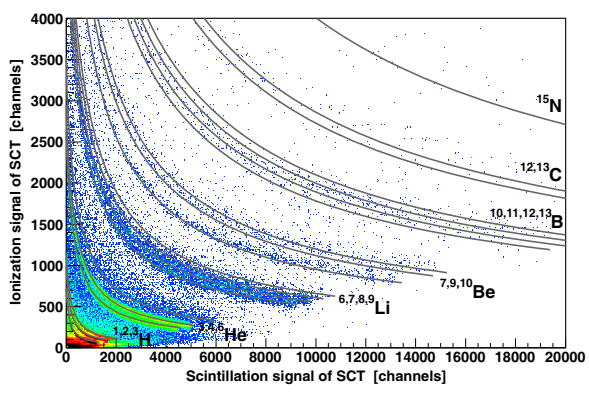

Figure 3: Identification spectrum for the SCT segment.

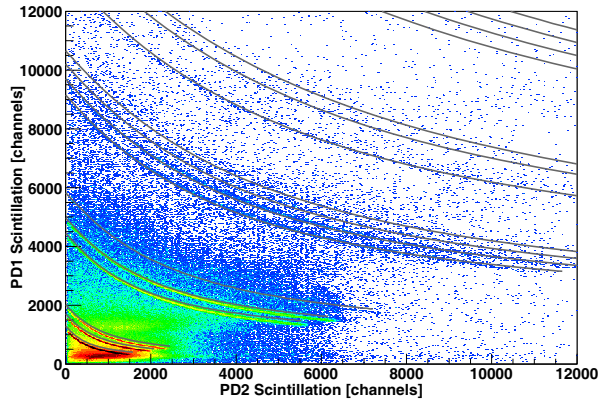

Figure 4: $\Delta E-E$ map for the thin vs thick $\operatorname{CsI}(\mathrm{Tl})$ crystals.

The isotopic resolution obtained using a single readout channel was found to compete very well with that obtained using the standard two channel readout (Fig. 3). The applied pulse shape analysis permitted also the identification of particles stopped in the first photodiode and the reduction of the identification threshold, due to the thickness of the first photodiode, by a factor of three. Thanks to the pulse shape analysis, it was also possible to obtain the ballistic deficit free amplitudes, which allowed for easy energy 
calibration and identification based on the predictions of the range-energy tables. For further analysis the parameters of the decomposed pulses were used in order to speed up the calculations.

In the identification maps, the isotopic lines are clearly visible, even though the background due to the secondary reactions in the long $\mathrm{CsI}(\mathrm{Tl})$ crystals is substantial (Fig. 4). In order to identify the events contributing to the background, a recognition procedure based on the self- organizing neural network has been developed [4]. This method groups together events with similar pulse shape descriptors. It allows to identify up to $90 \%$ of the background events. The efficiency of this method is presented in Fig. 5.

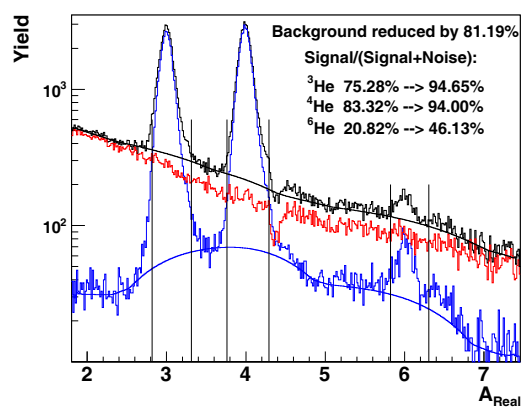

Figure 5: (Colour online) Helium isotopic distribution. Black line raw distribution, blue after background subtraction, red the background.

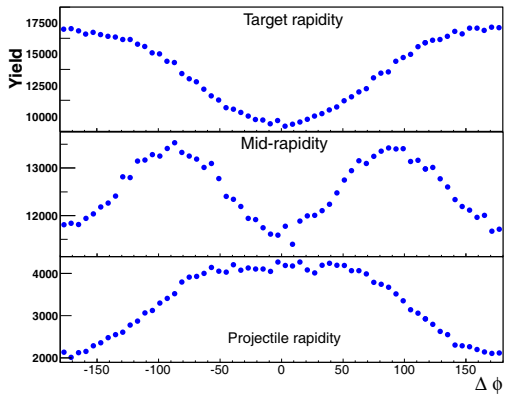

Figure 6: Azimuthal distributions of protons measured in three regions of the rapidity; $\mathrm{b}=(0-7.5$ $\mathrm{fm})$ for $\mathrm{Au}+\mathrm{Au}$ collisions at 400 $\mathrm{MeV} /$ nucleon.

The energy calibration was performed using the characteristic punchthrough points, curvatures of the $\Delta E-E$ lines, light-energy conversion formula and the ATIMA range-energy tables. Probabilities of the secondary reactions for light charged particles as a function of their range in the $\mathrm{CsI}(\mathrm{Tl})$ crystals was estimated using the Monte Carlo simulations within the GEANT4 environment. The data analysis was performed within the FairRoot framework.

The data measured with the KRATTA array in the ASY-EOS experiment was used to construct flow observables for light charged particles. The reaction plane orientation and the centrality of the collision were provided by the CHIMERA and ALADIN TOF Wall detectors. The reaction plane resolution corrections have been calculated according to [5].

Figure 6 shows the azimuthal distributions of protons measured with respect to the reaction plane in three rapidity regions. Target and projectile rapidity regions are dominated by the in-plane emissions, while the mid- 
rapidity range shows a strong squeeze-out signal.

Figure 7 presents the flow parameters $v_{1}$ and $v_{2}$ for LCPs as a function of the scaled center of mass rapidity measured for semi-central collisions with the KRATTA detector (circles) and the FOPI detector [6] (stars) in a common angular range (Fig. 2) The upper energy thresholds of KRATTA, associated with the $\mathrm{CsI}(\mathrm{Tl})$ crystal thickness, limit the range of the available rapidities. The data points are corrected for the secondary reaction background. The agreement between these two detectors and experiments is very satisfactory.

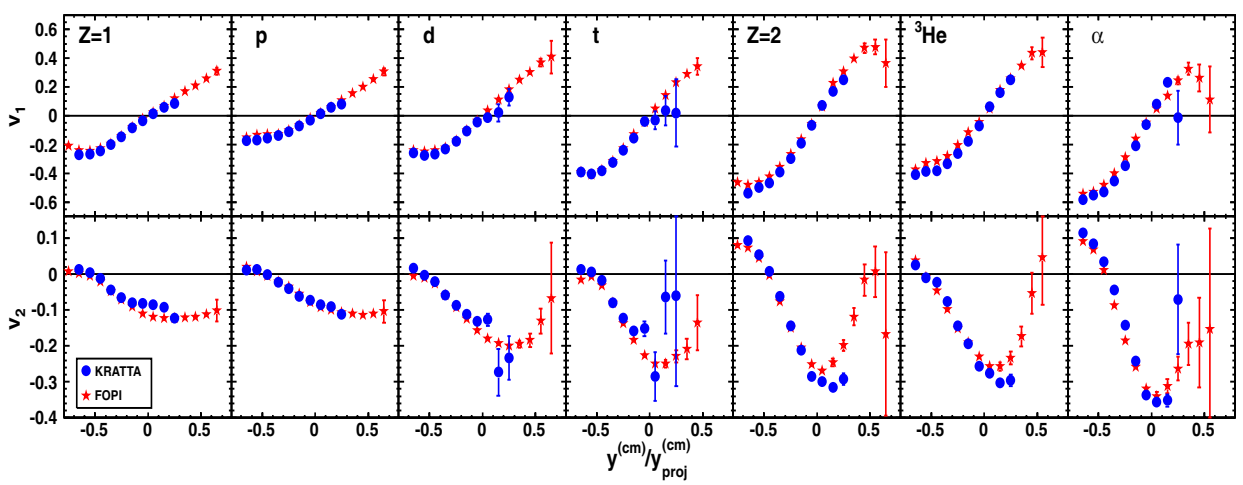

Figure 7: Flow of LCP measured with the KRATTA (circles) and FOPI (stars) detectors. Both measurements were performed in the polar angle range 38.5-56.5 deg and for the interval of reduced impact parameter range of $0.25-0.45$.

The KRATTA array, together with the CHIMERA and TOF-wall detectors, has proven its usefulness in flow measurements of light charged reaction products during the ASY-EOS experiment conducted at GSI. The preliminary comparison with the FOPI results measured for the same reaction in a different campaign shows a remarkable agreement and quality of the data. The results will be compared with UrQMD predictions in order to extract the information contained therein regarding the symmetry term in the nuclear equation of state.

\section{Acknowledgments}

The authors would like to thank W. Reisdorf for providing the FOPI flow data. One of us (S. K.) acknowledges the support by the Foundation for Polish Science - MPD program, co-financed by the European Union within the European Regional Development Fund. Work made possible through 
funding by Polish Ministry of Science and Higher Education under grant No. DPN/N108/GSI/2009.

\section{References}

[1] J. Łukasik et al., Nucl. Instr. Meth. A 709 (2013) 120128

[2] P. Russotto et al., Journal of Physics: Conference Series, 420. 5 (2013)

[3] G. Pasquali, et al., Nucl. Instr. Meth. A 301 (1991) 101

[4] S. Kupny, et al Acta Phys. Polon. B Suppl. 6, 1115-1119 (2013)

[5] J.-Y. Ollitrault arXiv:nucl-ex/9711003v2

[6] W. Reisdorf, FOPI Collaboration, private communication. 